
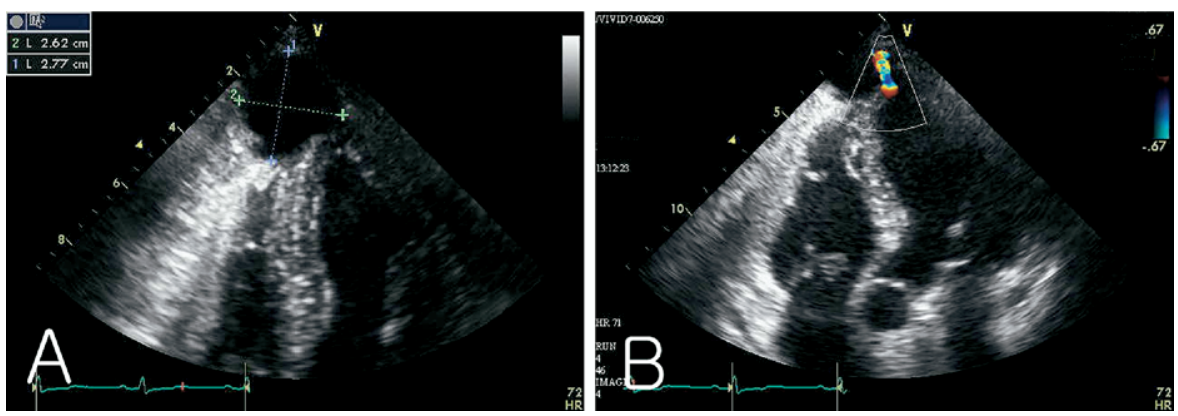

Figure 2. Postoperative echocardiogram. A, $2.62 \times 2.77-\mathrm{cm}$ pseudoaneurysm at the left ventricular apex. $B$, Flow through the neck of the pseudoaneurysm.

and abdominal pain, and pneumonia was diagnosed. During the discharge plan, sudden fever and chest pain developed in the patient. An electrocardiogram, a cardiac enzyme study, and a chest computed tomographic (CT) scan were performed to evaluate her symptoms. Other study results proved normal, but the chest CT scan showed type A aortic dissection. The ascending aorta and part of the arch were replaced by artificial grafts. Total circulatory arrest and selective cerebral perfusion were induced with axillary artery cannulation and a left ventricular venting catheter through the right superior pulmonary vein. There was severe pericardial adhesion, and a dissection of the adhesion was performed minimally only for the operation. The operation was uneventful, and 3 days later the patient was transferred to the general ward.

\section{Discussion}

Twenty days after the operation, a follow-up CT scan was performed, showing a pseudoaneurysm of the left ventricular apex (Figure 1). A follow-up echocardiogram also showed muscular disruption at the left ventricular apex with a $6.0 \mathrm{~m} / \mathrm{sec}$ flow through the neck of the pseudoaneurysm (Figure 2). Because there was no evidence of perioperative myocardial ischemia, such as an electrocardiogram change, a cardiac enzyme elevation, or a regional wall motion abnormality around the pseudoaneurysm, the pseudoaneurysm may have resulted from trauma to the left ventricular apex by the tip of the left ventricular venting catheter. It is likely that the left ventricular muscle damage during the operation was not seen because of the pericardial adhesion.

Despite our strong recommendations for surgery, the patient refused and was discharged. One month later, a follow-up echocardiogram showed no interval change.

\title{
Pseudoaneurysm of a saphenous bypass treated with covered endograft in the ascending aorta
}

\author{
Raphaël Coscas, MD, ${ }^{a}$ Isabelle Javerliat, MD, ${ }^{a}$ Arash M. Tofigh, MD, ${ }^{\text {a }}$ Thomas Hebert, MD, ${ }^{b}$ Mathieu Jablonski, MD,
} Philippe Cluzel, MD, ${ }^{b}$ Edouard Kieffer, MD, ${ }^{a}$ and Fabien Koskas, MD, PhDa Paris, France

\footnotetext{
From the Departments of Vascular Surgery ${ }^{\mathrm{a}}$ and Radiology, ${ }^{\mathrm{b}}$ Groupe Hospitalier Pitié-Salpétrière, Paris, France.

Received for publication Jan 21, 2007; revisions received Feb 6, 2007; accepted for publication Feb 19, 2007.

Address for reprints: Fabien Koskas, MD, PhD, Service de Chirurgie Vasculaire, Groupe hospitalier Pitié-Salpétrière, 47-83 Boulevard de l'Hôpital 75651 Paris Cedex 13 (E-mail: fabien.koskas@psl.ap-hop-paris.fr).

J Thorac Cardiovasc Surg 2007;134:230-2

$0022-5223 / \$ 32.00$

Copyright @ 2007 by The American Association for Thoracic Surgery doi:10.1016/j.jtcvs.2007.02.026
}

\section{Reference}

1. Mazzola A, Gregoriani R, Villani C, Di Eusanio M, Giancola R, Minuti $\mathrm{U}$, et al. Left ventricular pseudoaneurysm secondary to left ventricular venting through the right superior pulmonary vein. Ital Heart J. 2004; 5:881-2. 


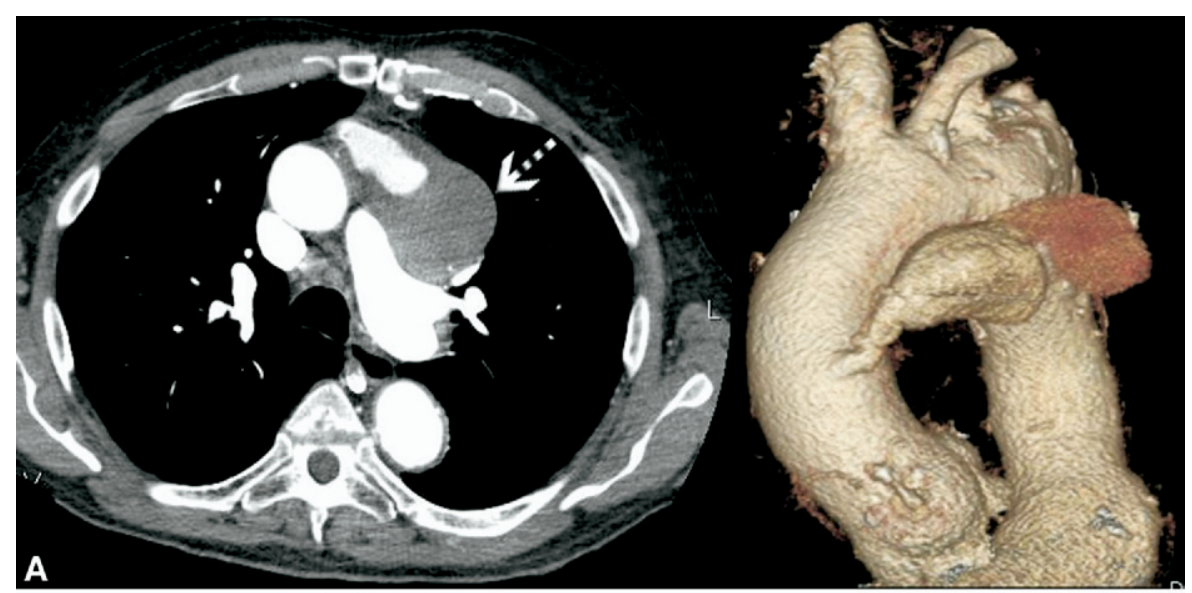

Figure 1. A, Computed tomographic scan with injection and electrocardiogram synchronization using a threedimensional reconstruction volume rendering technique. It revealed a 75-mm proximal saphenous vein graft pseudoaneurysm (arrow). B, Saphenous vein graft catheterization. It shows a proximal saccular dilatation of the saphenous vein graft (arrow). The bypass graft is not visible distal to the dilatation because it is occluded.


Figure 2. Computed tomographic scan with injection and electrocardiogram synchronization using a three-dimensional reconstruction volume rendering technique. Saphenous vein graft pseudoaneurysm is totally excluded /dotted arrow) and ascending aortic endograft is well positioned (full arrow). 
a pedicled left internal thoracic artery graft to the left anterior descending artery and a reversed saphenous vein graft to the first obtuse marginal branch. Since that time, the patient had remained free of symptoms.

On examination, the patient was afebrile with stable hemodynamics. He did not have any cardiac or pulmonary symptoms.

A computed tomographic scan revealed a $75-\mathrm{mm}$ proximal pseudoaneurysm resulting from a disruption of the aortic anastomosis (Figure 1,A). Cardiac catheterization showed a saccular dilatation of the saphenous vein graft. The bypass graft was occluded distal to the dilatation. The left internal thoracic artery graft was still patent (Figure 1,B).

Surgery was elected, but we felt that the risk of conventional surgery was prohibitive (American Society of Anesthesiologists score 3). After pluridisciplinary discussion, we decided to exclude this pseudoaneurysm by an endovascular procedure.

Nine days after admission, a covered endograft was positioned in the ascending aorta.

Our endograft was a homemade device built according to the method that we described earlier. ${ }^{2}$ In brief, the Gianturco arterial $\mathrm{Z}$ stent is an auto-expandable stainless steel structure (316L) made of a circular wire plied in a zigzag pattern (W. Cook Europe, Bjaeverskov, Denmark). Two Gianturco arterial Z stents were assembled with polyester ligatures (Cardioflon; Laboratoires Péters, Bobigny, France) and placed in a tube of uncrimped woven polyester (Twillweave; Vascutek, Incchinnan, Scotland) with a diameter of $36 \mathrm{~mm}$. The right common carotid artery was exposed through a transverse incision and punctured. Through the needle, a J-tipped 0.35 -inch guide wire was pushed into the aorta under fluoroscopy. A 5F sheath was then pushed over the wire. An Amplatz Superstiff guide wire (Boston Scientific, Natick, Mass) was then pushed into the sheath. Heparin, $1 \mathrm{mg} / \mathrm{kg}$, was given intravenously. Through a transverse arteriotomy centered by the puncture site, a 22F introducer (Keller-Timmermans; W. Cook Europe, Bjaeverskov, Denmark) was placed under fluoroscopic control over the guide into the ascending aorta. The endograft loaded into this introducer was then deployed by retraction of the sheath of this latter over its pusher.

The postoperative course was simple. No electrocardiographic change was noticed. Postprocedure cardiac enzymes were negative. On the fourth postoperative day, computed tomography confirmed the total exclusion of the pseudoaneurysm and the good position of the endograft (Figure 2). The patient was discharged on the sixth postoperative day.

The follow-up was uneventful (no cardiac symptoms, good recovery). Six months later, the patient remains free of symptoms. Computed tomography does not show any anomaly.

\section{Discussion}

This case demonstrates that selected cases of proximal aneurysm or pseudoaneurysm complicating coronary bypass with the saphenous vein are amenable to endovascular repair with endografts.

Severe dilatations complicating aorta-coronary bypass with the saphenous vein must be treated because of their potential complications, such as rupture and distal embolization.

These dilatations often occur in old and polypathologic patients. Moreover, when a surgical treatment is decided, redo surgery can be difficult and hazardous because of adhesions. Considering these problems, endovascular treatment was proposed in this indication. It was first described by Shapeero and associates ${ }^{3}$ in 1983. They treated a midgraft dilatation with occlusion therapy using coils. To our knowledge, 11 other cases of endovascular procedures have reported with good results. Several endovascular procedures have been used, such as coil occlusion therapy, covered stent in the saphenous vein graft, ${ }^{4}$ and Amplatzer vascular plug. ${ }^{5}$

In these cases, endovascular repair was performed only for body or distal saphenous vein graft dilatations.

In our case, conventional repair was not performed because of the patient's health status. As has been suggested for abdominal and thoracic aortic aneurysm, endografts could decrease morbidity and mortality in high-risk surgical patients. An endovascular procedure using an ascending aortic endograft seemed to be a reasonable option to exclude the pseudoaneurysm.

No suitable endograft was commercially available in our national market. The use of a commercially available endograft would have necessitated covering the supra-aortic trunks. We therefore preferred to use a specially tailored homemade endograft. $^{2}$

To our knowledge, our case is the first of a saphenous vein graft dilatation treated with an ascending aortic endograft.

\section{References}

1. Riahi M, Vasu CM, Tomatis LA, Schlosser RJ, Zimmerman G. Aneurysm of saphenous vein bypass graft to coronary artery. $J$ Thorac Cardiovasc Surg. 1975;70:358-9.

2. Koskas F, Cluzel P, Benhamou AC, Kieffer E. Endovascular treatment of aortoiliac aneurysms: made-to-measure stent-grafts increase feasibility. Ann Vasc Surg. 1999;13:239-46.

3. Shapeero LG, Guthaner DF, Swerdlow CD, Wexler L. Rupture of a coronary bypass graft aneurysm: CT evaluation and coil occlusion therapy. Am J Roentgenol. 1983;141:1060-2.

4. Mahy IR, Walton S. Successful treatment of false aneurysm of a saphenous vein bypass graft with fistula to the anterior chest wall using "covered" intracoronary stents. Heart. 1998;80:527-9.

5. Mylonas I, Sakata Y, Salinger MH, Feldman T. Successful closure of a giant true saphenous vein graft aneurysm using the Amplatzer vascular plug. Catheter Cardiovasc Interv. 2006;67:611-6. 